\title{
Modelling, validation, and control of an industrial fuel gas blending system ${ }^{\text {is }}$
}

\author{
C.J. Muller ${ }^{\mathrm{a}, \mathrm{b}}$, I.K. Craig ${ }^{\mathrm{b}, *}$, N.L. Ricker ${ }^{\mathrm{c}}$ \\ a Sasol Solvents RSA, Sasolburg, South Africa \\ ${ }^{\mathrm{b}}$ Department of Electrical, Electronic, and Computer Engineering, University of Pretoria, Pretoria, South Africa \\ ${ }^{c}$ Department of Chemical Engineering, University of Washington, Seattle, USA
}

\section{A R T I C L E I N F O}

\section{Article history:}

Received 21 January 2011

Received in revised form 10 March 2011

Accepted 2 April 2011

Available online $\mathrm{xxx}$

\section{Keywords:}

Dynamic model

Model-based control

State-space model

Validation

Real-time optimisation

MPC

RTO

\begin{abstract}
A B S T R A C T
In industrial fuel gas preparation, several compositional properties must be controlled within specified limits. This allows client plants to burn the gas safely and with consistent heat production. The variables to be controlled are the higher heating value (HHV), Wobbe index (WI), flame speed index (FSI), and header pressure. A plant in which six feed gasses are blended is considered. Four of the feeds are well-defined makeup streams (costly but always available) and the other two are byproducts that would otherwise be flared. The six feed rates comprise the manipulated variables (MVs) used to regulate the four controlled variables (CVs) while minimising the cost of the gas blend. The control system must compensate for feed composition and fuel gas demand variability. The development and validation of an industrial fuel gas header model is described, followed by a simulation study comparing three Model Predictive Control (MPC) strategies. It is shown that when iterative linearisation is used to update the prediction model and real-time optimisation (RTO) is used to update the CV and MV targets used in the MPC cost function, the plant is driven reliably to the optimal steady-state.
\end{abstract}

(c) 2011 Elsevier Ltd. All rights reserved.

\section{Introduction}

The fuel gas used in industrial plants must satisfy several compositional constraints in order for the gas to burn safely, reliably, and produce the expected heat output. Key properties include the higher heating value (HHV or gross calorific value) [1], Wobbe index (WI), and flame speed index (FSI, using Weaver's flame speed factor) [2], all of which must stay within prescribed ranges. The fuel gas header pressure must also be kept within a target range.

The industrial syngas production process considered here results in two byproducts (tail gases) that may be burned to satisfy heating needs elsewhere in the complex. Unused tail gases are flared, so there is a strong incentive to maximise their utilisation as fuels. Their compositional properties are unfavourable, however, and they must be blended with makeup gases in order to satisfy the constraints mentioned previously. In the syngas complex, four makeup gases are used, leading to a total of six blended gas streams, the rates of which are termed the manipulated variables (MVs) in the present context.

\footnotetext{
A preliminary version of this article was submitted to the 18th IFAC World Congress, Milan, 2011.

* Corresponding author at: Department of Electrical, Electronic, and Computer Engineering, University of Pretoria, Pretoria, South Africa.

Tel.: +27 12420 2172; fax: +27 123625000 .

E-mail addresses: nelis.muller@sasol.com, nelismuller@tuks.co.za (C.J. Muller), icraig@postino.up.ac.za (I.K. Craig), ricker@u.washington.edu (N.L. Ricker).
}

Tail gas availabilities and compositions vary significantly as a function of time. The primary goal of fuel gas blending is to compensate for such disturbances by adjusting the MVs so as to satisfy continuously the HHV, WI, FSI, and header pressure constraints. The system must also respond to changes in fuel gas demand. Doing so manually is a challenging task, even for the most experienced operators. A secondary goal is to minimise the cost of the blended gas. Under manual control, this is often much higher than the optimum.

There are many publications on fuel gas properties and their impact on combustion, but little regarding advanced control and economic optimisation of fuel gas blending (although industrial applications exist). There are, however, publications regarding the control of liquid blends. For example, Chèbre et al. [3] describe continuous and batch blending of liquid fuels to produce mixtures with prescribed properties while minimising the production cost. Their algorithm is essentially a specialised version of Model Predictive Control (MPC). A key aspect is the use of an estimator to determine uncertain parameters needed to predict the impact of MV adjustments on blend properties.

There are two important differences between this and the problem considered in the present work. On one hand, the blended gases behave as nearly ideal mixtures and their combustion properties are known functions of composition. Moreover, periodic measurements of the feed gas compositions are available. Thus, prediction of the impact of MV adjustments on blend properties is easier than in liquid blending. On the other hand, the gas blending system's mean residence time is of order $2-4 \mathrm{~min}$, whereas that in a typical liquid blending system is hours or longer. Thus, the gas blending system 


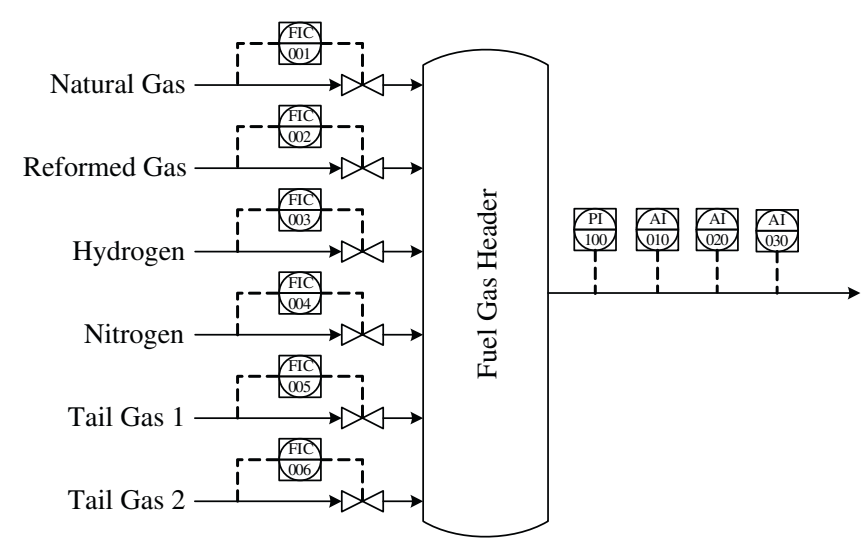

Fig. 1. Process diagram of blending header.

places more emphasis on measurement speed and frequency, and requires relatively frequent MV adjustments.

Ratio control would appear to be an obvious automation strategy, but the tail gas compositional variability is such that the use of constant ratios would lead to constraint violations. One could add a multi-loop supervisory layer to adjust the ratios, but compensation for changes in demand and availability would require a complex configuration of interconnected PID controllers and overrides.

As suggested by Chèbre et al. [3] and others, MPC allows one to formulate a blending control problem directly. It also offers the possibility of simultaneous economic optimisation. The primary contribution of this application paper is to determine the extent to which a standard MPC can achieve the technical and economic objectives in fuel gas blending. Several MPC formulations are tested in order to evaluate and minimise the impact of process nonlinearities, and to improve the economic performance.

\section{Process overview}

Fig. 1 shows a process diagram of the system. Although the header is depicted as a vessel, it is actually a piping network of fixed volume. The flow rates are high so it is assumed that turbulent flows facilitate perfect mixing such that the composition of the exit stream equals the header composition (which is assumed to be uniform within the header). Six gas streams enter the fuel gas header (shown with their fictional tag names in Fig. 1). These six feed streams are natural gas (NG), reformed gas (RG, a hydrogen to CO ratio of between $1.8: 1$ and $2: 1)$, hydrogen $\left(\mathrm{H}_{2}\right)$, nitrogen $\left(\mathrm{N}_{2}\right)$, Tail Gas $1\left(\mathrm{TG}_{1}\right)$, and Tail Gas $2\left(\mathrm{TG}_{2}\right)$. The first four are make-up streams whereas the two tail gases are wild streams, varying in availability and composition. The six feeds must be mixed in correct ratios and quantities to control the output composition and pressure. Table 1 shows the specified ranges for the CVs.

The NG, RG, and $\mathrm{N}_{2}$ streams have costs associated with them whereas the $\mathrm{H}_{2}$ and tail gas streams would otherwise be flared and thus have zero cost. Therefore, the use of the NG, RG, and $\mathrm{N}_{2}$ streams should be minimised whereas the use of the tail gas streams and $\mathrm{H}_{2}$ should be maximised subject to their availabilities. Natural gas is used continuously to increase the calorific value to specification.

Table 1

Controlled variable ranges.

\begin{tabular}{llll}
\hline Controlled variable & Abbr. & Range & Units \\
\hline Higher heating value & HHV & $16.5-18$ & $\mathrm{MJ} / \mathrm{Nm}^{3}$ \\
Wobbe index & WI & $25-27$ & $\mathrm{MJ} / \mathrm{Nm}^{3}$ \\
Flame speed index & FSI & $39-46$ & - \\
Pressure & P & $2000-2200$ & $\mathrm{kPa}$ \\
\hline
\end{tabular}

Table 2

Typical inlet compositions ( $\mathrm{mol} \%$ ).

\begin{tabular}{lrrrrrr}
\hline & $\mathrm{NG}$ & \multicolumn{1}{c}{$\mathrm{RG}$} & $\mathrm{H}_{2}$ & $\mathrm{~N}_{2}$ & $\mathrm{TG}_{1}$ & \multicolumn{1}{c}{$\mathrm{TG}_{2}$} \\
\hline $\mathrm{CH}_{4}$ & 91.1 & 1.5 & - & - & 5.5 & 15.0 \\
$\mathrm{C}^{+}+$ & 6.8 & 0.0 & - & - & 1.0 & 1.0 \\
$\mathrm{H}_{2}$ & 0.0 & 62.0 & 100 & - & 62.0 & 57.0 \\
$\mathrm{~N}_{2}$ & 1.5 & 0.5 & - & 100 & 2.5 & 6.0 \\
$\mathrm{CO}$ & 0.0 & 31.0 & - & - & 26.0 & 13.0 \\
$\mathrm{CO}_{2}$ & 0.6 & 5.0 & - & - & 3.0 & 8.0 \\
$\mathrm{HHV}$ & 43.02 & 11.78 & 12.10 & 0.0 & 13.96 & 15.39 \\
$W I$ & 52.62 & 17.87 & 45.73 & 0.0 & 21.60 & 22.92 \\
\hline
\end{tabular}

Nitrogen is needed only when the FSI is too high. Reformed gas is used when the tail gas streams are not available.

Important disturbances include changes in feed stream composition (especially the two tail gases) and total fuel gas demand (i.e. the discharge flow rate from the header). Table 2 gives the typical compositions and feed stream characteristics. The HHV, WI, and FSI are functions of the molar composition of the fuel gas (see Section 3).

All gas flow rates as well as the HHV, WI, and header pressure are measured continuously and sampled every 20 s. Feed and blended fuel gas compositions are measured by mass spectrometry on a 10 -min cycle. The fuel gas FSI is re-calculated each time a new composition measurement becomes available, i.e., every $10 \mathrm{~min}$.

As mentioned previously, for a typical fuel gas demand of $30 \mathrm{kN} \mathrm{m}^{3} / \mathrm{h}$, the gas residence time is of order four minutes and transport delays are negligible. The dominant delays are the analyser measurement latencies, which are nearly constant.

\section{Modelling}

The header can be modelled with six states (the molar quantity of each of the six chemical components in the header), six MVs (the volumetric flow rates of the six inlet streams), and four CVs (HHV, FSI, WI, and pressure). The state equations are given by

$\dot{N}_{f g, i}=u_{i}-y_{f g, i} \cdot u_{T}$

where $i=1-6, N_{f g, i}$ is the number of moles of component $i$ in the header, $u_{i}$ is the total molar flow of component $i$ entering the header (summed over all inlet steams), $u_{T}$ is the total molar discharge rate from the header, and $y_{f g, i}$ is the molar fraction of component $i$ in the header. The actual MVs are volumetric flow rates measured in $\mathrm{kN} \mathrm{m}^{3} / \mathrm{h}$, which are related to $u_{i}$ as follows:

$u_{i}=44.64 \sum_{j=1}^{6} y_{F_{j}, i} \cdot F_{j}$

for $i=1-6$ where $F_{j}$ is the volumetric flow rate of the $j$ th feed stream $\left(\mathrm{kN} \mathrm{m}^{3} / \mathrm{h}\right)$, and $y_{F_{j}, i}$ is the molar fraction of component $i$ in inlet stream $j$. The $j$ index refers to the sequence shown in Fig. 1.

The outputs are calculated according to the molar fractions of the components in the system (and the total number of moles in the case of pressure). The output calculations are

$$
\begin{aligned}
& H H V_{f g}=\sum_{i=1}^{6} H H V_{i} \cdot y_{f g, i} \\
& W I_{f g}=\frac{H H V_{f g}}{\sqrt{\rho_{f g}}}
\end{aligned}
$$


Table 3

Component characteristics.

\begin{tabular}{lcclrrr}
\hline & $H H V$ & $W I$ & SG & MWt & \multicolumn{1}{c}{$A$} & $s$ \\
\hline $\mathrm{CH}_{4}$ & 37.78 & 50.72 & 0.557 & 16.04 & 9.55 & 148 \\
$\mathrm{C} 2-\mathrm{C} 6$ & 126.50 & 87.62 & 2.018 & 58.12 & 31.00 & 514 \\
$\mathrm{H}_{2}$ & 12.10 & 45.88 & 0.069 & 2.02 & 2.39 & 339 \\
$\mathrm{~N}_{2}$ & - & - & 0.973 & 28.02 & - & - \\
$\mathrm{CO}$ & 11.97 & 12.17 & 0.968 & 28.01 & 2.39 & 61 \\
$\mathrm{CO}_{2}$ & - & - & 1.528 & 44.01 & - & - \\
\hline
\end{tabular}

$F S I_{f g}=\frac{\sum_{i=1}^{6} y_{f g, i} \cdot S_{i}}{\sum_{i=1}^{6} y_{f g, i} \cdot A_{i}+5 \sum_{j=1}^{2} n_{f g, j}-18.8 x_{\mathrm{O}_{2}}+1}$

$P=\frac{N_{T} R T}{V}$

where $s_{i}$ is the flame speed factor for component $i, A_{i}$ is the molar stoichiometric air demand factor (for total combustion) for component $i, n_{f g, j}$ is the molar fraction of inert component $j$ in the fuel gas, $x_{\mathrm{O}_{2}}$ is the mole fraction of oxygen in the gas (usually zero in this application), $N_{T}$ is the total number of moles in the header, $R=8.314$ is the gas constant, $T$ is the header temperature (Kelvin) and $V$ is the header volume $\left(\mathrm{m}^{3}\right.$, estimated at $\left.100 \mathrm{~m}^{3}\right)$. The fuel gas specific gravity, $\rho_{f g}$, is calculated as

$\rho_{f g}=\frac{\sum_{i=1}^{6} M W t_{i} \cdot y_{f g, i}}{M W t_{\text {air }}}$

where $M W t_{i}$ is the molar weight of component $i$ and $M W t_{\text {air }}=28.8$ is the standard molar weight of air. Table 3 lists some characteristics of the components [1].

\subsection{Model validation}

The integrity of the process model must be determined in order to support the validity of the simulation study. For the validation, a period of operation was identified in which all the flow measurements were reliable (either zero or greater than the turn-down of the transmitters). The inlet flow rates, feed stream compositions, and header discharge rate were used as verification data and the simulation output data compared to the plant measurements (the system is at ambient temperature and diurnal temperature variations have a negligible impact). The initial model states were the steady values corresponding to the average feed flow rates and compositions.

The sampling rate for the plant data is $20 \mathrm{~s}(1 / 180 \mathrm{~h})$. Analyser dead times (in measurements of HHV, WI, and FSI) were initially estimated at 2 min each and the volume at $100 \mathrm{~m}^{3}$ (based on the rate of change in pressure with regard to change in the inlet flow rates). These parameters were adjusted recursively to improve the fit with regard to the correlation coefficients obtained. The final dead-time estimates were $20 \mathrm{~s}$ for HHV, $1 \mathrm{~min}$ for WI, and $20 \mathrm{~s}$ for FSI. There was no need to adjust the total volume, which was kept at $100 \mathrm{~m}^{3}$.

As shown in Figs. 2-4, the model's open-loop predictions of HHV, WI, and FSI track the observed trends rather well. Computed correlation coefficients for a validation period of $18 \mathrm{~h}$ are shown in Table 4 . Some factors contributing to the discrepancies between the plant data and the model include infrequent feed stream and fuel gas composition measurements, errors in feed flow measurements (especially when close to the turn-downs of the flow transmit-

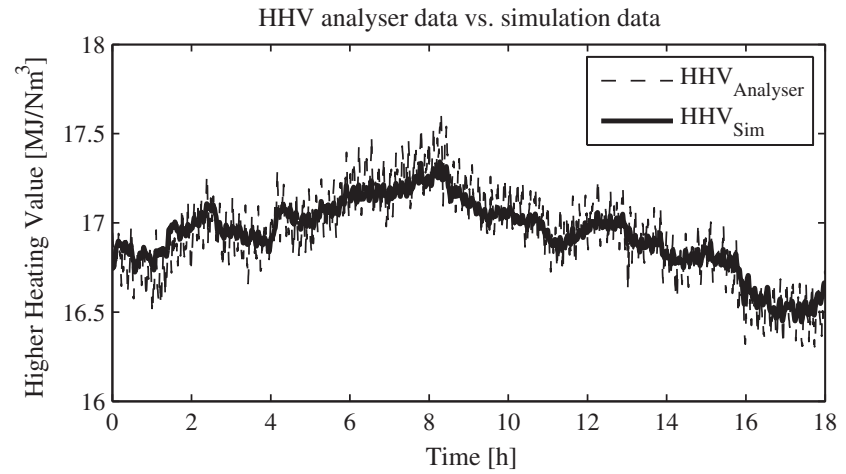

Fig. 2. HHV analyser data versus simulation data.

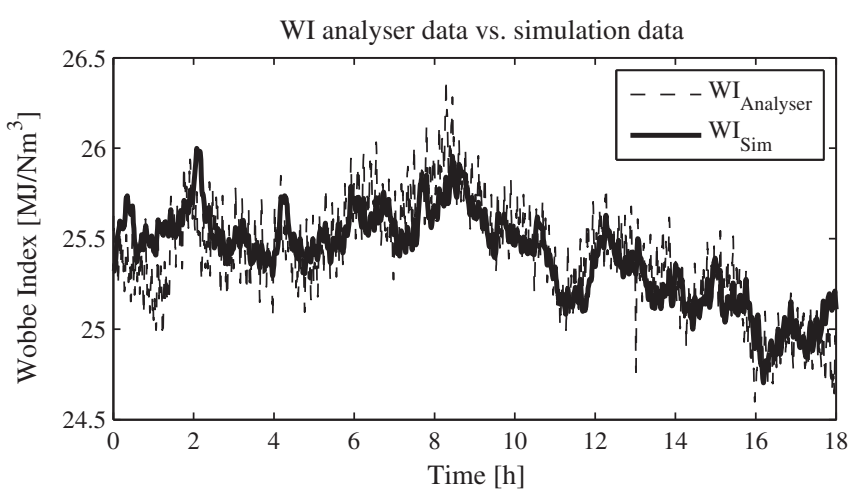

Fig. 3. WI analyser data versus simulation data.

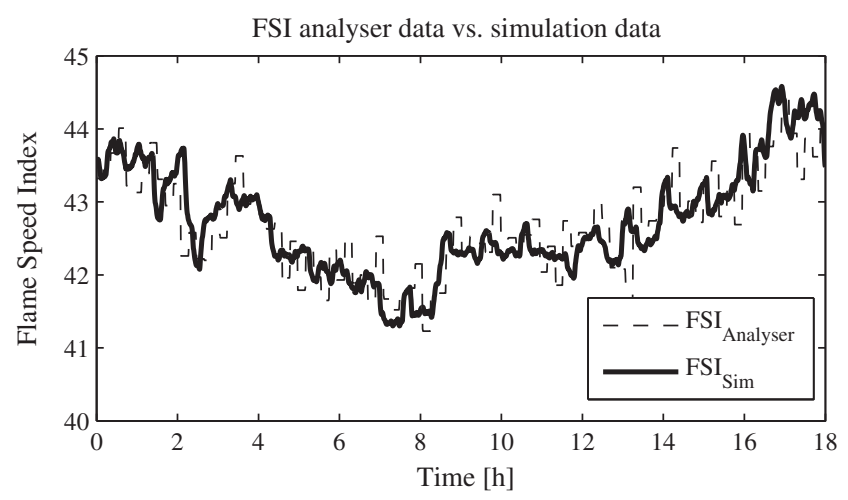

Fig. 4. FSI analyser data versus simulation data.

ters), and interpolation adjustments made when the plant's data historian recorded the data.

The presence of feedback control on the header pressure for all plant data complicates the validation of the pressure model. Feedback can introduce non-causal effects from input to output (for example an operator who anticipates an event and compensates for it before it actually occurs) which complicates the validation [12]. The model's pressure variations are, however, based on well established physical principles and will be assumed adequate for the purposes of this simulation study.

Table 4

Correlation coefficients for output data.

\begin{tabular}{ll}
\hline Data set & Correlation coefficient (\%) \\
\hline HHV & 93.8 \\
WI & 84.8 \\
FSI & 83.1 \\
\hline
\end{tabular}




\subsection{Linearising the model for control purposes}

If the feed compositions, header pressure, and fuel gas demand are constant, the state equation is linear, and the response of each $y_{f g, i}$ to a change in the corresponding $u_{i}$ is first-order. This is not the case in general, however. Also, the relationships between the states and the WI and FSI are nonlinear. Therefore, a linear model was derived as the starting point for controller design.

Several types of LTI (Linear Time-Invariant) models were considered. As would often be the case in practice, first order plus dead time transfer function models were selected for HHV, WI, and FSI and an integrator was used to model the pressure dynamics. The linearisation was performed around an operating point of [HHV, WI, FSI, P] $=[16.75,25.32,43.47,2085]$ which is a typical operating region for the plant. The resulting nominal model appears in Table 5, where the time unit is hours.

\section{Iterative linearisation}

One of the MPC strategies to be described later adjusts its model gains to adapt to measured feed composition variations. The expected steady state values of the HHV, WI, and FSI can be calculated as a function of the MVs and the feed stream compositions. Thus, for a given set of feed stream compositions, one can compute the steady-state gains of these CVs with respect to the MVs. The gain calculations are described in the next sections [4-6].

\subsection{Heating value}

The fuel gas heating value is calculated as

$H H V_{f g}=\frac{\sum_{i=1}^{6} F_{i} \cdot H H V_{F_{i}}}{F_{T}}$

where $F_{i}$ and $H H V_{F_{i}}$ are the volumetric flow rate $\left(\mathrm{kN} \mathrm{m}^{3} / \mathrm{h}\right)$ and heating value $\left(\mathrm{MJ} / \mathrm{Nm}^{3}\right)$ of the $i$ th inlet stream and $F_{T}=\sum_{6}^{i=1} F_{i}$ is the total inlet volumetric flow rate. The gains are then calculated as

$\frac{\partial H H V_{f g}}{\partial F_{i}}=\frac{H H V_{F_{i}}-H H V_{f g}}{F_{T}}$

\subsection{Wobbe index}

The Wobbe index is calculated as (same as Eq. (4))

$W I_{f g}=\frac{H H V_{f g}}{\sqrt{\rho_{f g}}}$

where $H H V_{f g}$ is given in Eq. (8) and $\rho_{f g}$ is the relative density of the fuel gas, calculated with regard to inlet flow rates as

$\rho_{f g}=\frac{\sum_{i=1}^{6} F_{i} \cdot \rho_{F_{i}}}{F_{T}}$

where $\rho_{F_{i}}$ is the relative density of inlet gas $i$. Taking the derivative of $W I_{f g}$ with regard to $F_{i}$ gives

$\frac{\partial W I_{f g}}{\partial F_{i}}=\frac{1}{\sqrt{\rho_{f g}}} \cdot \frac{\partial H H V_{f g}}{\partial F_{i}}-\frac{H H V_{f g}}{2 \cdot \rho_{f g}^{1.5}} \cdot \frac{\partial \rho_{f g}}{\partial F_{i}}$

with $\frac{\partial H H V_{f g}}{\partial F_{i}}$ given in Eq. (9) and

$\frac{\partial \rho_{f g}}{\partial F_{i}}=\frac{\rho_{F_{i}}-\rho_{f g}}{F_{T}}$

\subsection{Flame speed index}

The flame speed formula is shown in Eq. (5), with the values for $A_{i}$ and $s_{i}$ given in Table 3. To calculate the FSI in terms of the inlet volumetric flow rates, the molar components in the fuel gas are calculated using

$y_{f g, i}=\frac{\sum_{j=1}^{6} F_{j} \cdot y_{F j, i}}{F_{T}}$

where $y_{F j, i}$ is the molar fraction of component $i$ in inlet stream $j$. The derivative can then be determined as

$\frac{\partial F S I_{f g}}{\partial F_{i}}=\sum_{x=1}^{4} \frac{\partial F S I_{f g}}{\partial y_{f g, x}} \cdot \frac{\partial y_{f g, x}}{\partial F_{i}}+\sum_{k=1}^{2} \frac{\partial F S I_{f g}}{\partial n_{f g, k}} \cdot \frac{\partial n_{f g, k}}{\partial F_{i}}$

where $y_{f g, x}$ refers to the molar fraction of combustible component $x$ in the fuel gas and $n_{f g, k}$ is the molar fraction of inert component $k$ in the fuel gas. The individual terms in Eq. (15) are given by

$$
\begin{aligned}
\frac{\partial F S I_{f g}}{\partial y_{f g, x}} & =\frac{s_{x}-A_{x} \cdot F S I_{f g}}{\sum_{i=1}^{2} y_{f g, i} \cdot A_{i}+5 \sum_{j=1}^{2} n_{f g, j}-18.8 x_{\mathrm{O}_{2}}+1} \\
\frac{\partial y_{f g, x}}{\partial F_{i}} & =\frac{y_{F_{i}, x}-y_{f g, x}}{F_{T}} \\
\frac{\partial F S I_{f g}}{\partial n_{f g, k}} & =\frac{-5 . F S I_{f g}}{\sum_{i=1} y_{f g, i} \cdot A_{i}+5 \sum_{j=1}^{2} n_{f g, j}-18.8 x_{O_{2}}+1} \\
\frac{\partial n_{f g, k}}{\partial F_{i}} & =\frac{n_{F_{i}, k}-n_{f g, k}}{F_{T}}
\end{aligned}
$$

\section{Control}

\subsection{MPC overview}

Since the early description of MPC (Model Predictive Control) in the late 1970s by [7] and its application in the refining industry by Shell Oil [8], significant attention has been given to the development of this powerful advanced control technique. MPC is a model-based control strategy that uses a dynamic model of a system to predict its future behaviour and then calculate the optimal control moves to minimise a cost function, subject to constraints [9]. Advantages of MPC include flexibility in formulating the objective function and defining the process model, the ability to include equality and inequality constraints directly in the control law, accommodation of multivariable systems, and the possibility of dealing with large disturbances quickly (due to its feed forward control capability). The main drawbacks in using MPC are the computational burden associated with it (especially when considering large systems and large control and prediction horizons) and the need for a reliable model of the process [10].

There are many MPC variants, differing mainly in the type of model used, the form of the cost function and constraints, and the way the controller handles noise and disturbances [10]. An overview of commercial MPC technologies can be found in [11].

\subsection{Nominal MPC design for the fuel gas blending process}

The focus of the paper is a solution implementable using standard tools readily available to the process industries (see e.g. [11]) As a first step, the nominal LTI model described in Section 3.2 
Table 5

Linearised model matrix.

\begin{tabular}{|c|c|c|c|}
\hline & NG & RG & $\mathrm{H}_{2}$ \\
\hline HHV & $\frac{24.61}{s+28.62} e^{-s / 180}$ & $\frac{-4.42}{s+23.12} e^{-S / 180}$ & $\frac{-5.04}{S+26.44} e^{-S / 180}$ \\
\hline$W I$ & $\frac{30.73}{s+28.69} e^{-s / 60}$ & $\frac{-6.23}{s+23.39} e^{-s / 60}$ & $\frac{2.25}{s+26.29} e^{-s / 60}$ \\
\hline FSI & $\frac{-59.21}{s+28.57} e^{-s / 180}$ & $\frac{11.05}{s+22.92} e^{-S / 180}$ & $\frac{32.26}{s+27.14} e^{-s / 180}$ \\
\hline \multirow[t]{2}{*}{$P$} & $\frac{1120}{s}$ & $\frac{1120}{s}$ & $\frac{1120}{s}$ \\
\hline & $\mathrm{N}_{2}$ & $\mathrm{TG}_{1}$ & $\mathrm{TG}_{2}$ \\
\hline HHV & $\frac{-13.27}{s+23.09} e^{-s / 180}$ & $\frac{-3.31}{s+25.78} e^{-s / 180}$ & $\frac{-1.66}{s+22.64} e^{-s / 180}$ \\
\hline$W I$ & $\frac{-30.42}{s+23.00} e^{-s / 60}$ & $\frac{-4.04}{s+25.62} e^{-s / 60}$ & $\frac{-2.45}{s+22.85} e^{-S / 60}$ \\
\hline FSI & $\frac{-33.59}{s+23.00} e^{-s / 180}$ & $\frac{10.40}{s+26.72} e^{-s / 180}$ & $\frac{2.23}{s+22.17} e^{-s / 180}$ \\
\hline$P$ & $\frac{1120}{s}$ & $\frac{1120}{s}$ & $\frac{1120}{s}$ \\
\hline
\end{tabular}

Table 6

Relative costs of inlet streams $\left[\operatorname{cost} / \mathrm{kN} \mathrm{m}^{3}\right]$.

\begin{tabular}{ll}
\hline Feed stream & Relative cost \\
\hline $\mathrm{NG}$ & 0.678 \\
$\mathrm{RG}$ & 0.254 \\
$\mathrm{H}_{2}$ & 0 \\
$\mathrm{~N}_{2}$ & 0.068 \\
$\mathrm{TG}_{1}$ & 0 \\
$\mathrm{TG}_{2}$ & 0 \\
\hline
\end{tabular}

was used to define a base-case MPC. The specific MPC algorithm used was the MATLAB Model Predictive Control Toolbox [12]. The nominal model presents no unusual characteristics, and a standard finite-horizon cost function was expected to provide adequate nominal performance and stability.

The MPC sampling period was fixed at the plant sampling period: $20 \mathrm{~s}$. The average settling time for the HHV, WI, and FSI is about $12 \mathrm{~min}$. The initial values for the prediction and control horizons were chosen according to the guidelines in [13], which suggested a prediction horizon of 44 control intervals with 5 control moves. These values were changed by trial and error (to prevent the controller from being too aggressive), arriving at a final prediction horizon of 39 intervals and 3 control moves. Control horizon blocking was used to distribute the control moves over the prediction horizon (a block is one or more successive sampling periods over which the MVs are kept constant [12]). The final block durations were 2,6 , and 31 intervals.

In an attempt to include economic considerations, MV weights were chosen in proportion to the feed costs (given in Table 6) with identical rate weights (see Table 7). According to the standard cost function, the MV weights penalise the deviation of each MV from a specified target value. The base-case MPC used constant MV targets of [0 0503030$]$.

The CVs were weighted to penalise deviations from nominal targets, which were chosen to be mid-range (i.e. $H H V_{\text {nominal }}=17.25$, $W I_{\text {nominal }}=26, F S I_{\text {nominal }}=42.5$, and $P_{\text {nominal }}=2100$ ). The numerical values were chosen according to priority of the CVs where the highest priority is given to HHV and the lowest to pressure (see Table 8). Note that this discourages the controller from driving the CVs to their limits, which is often necessary for economic optimisation.

Table 7

Weights on MVs.

\begin{tabular}{lcl}
\hline MV & Weight & Rate weight \\
\hline $\mathrm{NG}$ & 67 & 1 \\
$\mathrm{RG}$ & 25 & 1 \\
$\mathrm{H}_{2}$ & 0 & 1 \\
$\mathrm{~N}_{2}$ & 7 & 1 \\
$\mathrm{TG}_{1}$ & 0 & 1 \\
$\mathrm{TG}_{2}$ & 0 & 1 \\
\hline
\end{tabular}

Table 8

Weights on CVs.

\begin{tabular}{lc}
\hline CV & Weight \\
\hline HHV & 100 \\
WI & 80 \\
FSI & 70 \\
P & 20 \\
\hline
\end{tabular}

The rationale was that the base-case MPC, which uses an LTI model, might not be accurate enough to allow operation near constraint boundaries. The CV weights were adjusted by trial to arrive at the values shown in Table 8.

Finally, hard bounds were defined for the MVs and soft bounds were defined for the CVs. The MV lower bounds were zero and their upper bounds were the availabilities of each MV (see Table 9). The CV bounds were the ranges shown in Table 1.

To summarise, the base-case MPC formulation was

$\sum_{i=0}^{p-1}\left(S_{y, i}(k)+S_{\Delta u, i}(k)+S u, i(k)\right)+\rho_{\varepsilon} \varepsilon^{2}$

with

$S_{y, i}(k)=\sum_{j=1}^{n_{y}}\left|w_{j}^{y}\left(y_{j}(k+i+1 \mid k)-r_{j}(k+i+1)\right)\right|^{2}$

$S_{\Delta u, i}(k)=\sum_{j=1}^{n_{u}}\left|w_{j}^{\Delta u} \Delta u_{j}(k+i \mid k)\right|^{2}$

$S_{u, i}(k)=\sum_{j=1}^{n_{u}}\left|w_{j}^{u}\left(u_{j}(k+i \mid k)-u_{j_{\text {target }}}(k+i)\right)\right|^{2}$

subject to

$u_{j_{\min }}(i)-\varepsilon V_{j_{\min }}^{u}(i) \leq u_{j}(k+i \mid k) \leq u_{j_{\max }}(i)+\varepsilon V_{j_{\max }}^{u}(i)$

$\Delta u_{j_{\min }}(i)-\varepsilon V_{j_{\min }}^{\Delta u}(i) \leq \Delta u_{j}(k+i \mid k) \leq \Delta u_{j_{\max }}(i)+\varepsilon V_{j_{\max }}^{\Delta u}(i)$

$y_{j_{\min }}(i)-\varepsilon V_{j_{\min }}^{y}(i) \leq y_{j}(k+i+1 \mid k) \leq y_{j_{\max }}(i)+\varepsilon V_{j_{\max }}^{y}(i)$

Table 9

Limits on MVs $\left[\mathrm{kN} \mathrm{m}^{3} / \mathrm{h}\right]$.

\begin{tabular}{llcl}
\hline MV & Low limit & High limit & Units \\
\hline $\mathrm{NG}$ & 0 & 15 & $\mathrm{kN} \mathrm{m}^{3} / \mathrm{h}$ \\
$\mathrm{RG}$ & 0 & 20 & $\mathrm{kN} \mathrm{m}^{3} / \mathrm{h}$ \\
$\mathrm{H}_{2}$ & 0 & 5 & $\mathrm{kN} \mathrm{m}^{3} / \mathrm{h}$ \\
$\mathrm{N}_{2}$ & 0 & 5 & $\mathrm{kN} \mathrm{m}^{3} / \mathrm{h}$ \\
$\mathrm{TG}_{1}$ & 0 & 30 & $\mathrm{kN} \mathrm{m}^{3} / \mathrm{h}$ \\
$\mathrm{TG}_{2}$ & 0 & 30 & $\mathrm{kN} \mathrm{m}^{3} / \mathrm{h}$ \\
\hline
\end{tabular}


$i=0, \ldots, p-1$

$u(k+h \mid k)=0, \quad h=m, \ldots, p-1$

$\varepsilon \geq 0$

where $\Delta u(k+i \mid k)$ is the MV change vector at time $k+i$ based on the information available at time $k, m$ is the control horizon, $p$ is the prediction horizon, $\varepsilon$ is a slack variable, $\rho_{\varepsilon}$ is the weight on the slack variable, $r(k)$ is the CV reference vector at time $k, w_{j}^{y}, w_{j}^{\Delta u}$, and $w_{j}^{u}$ are weighting matrices for the CVs, MV rates (or increments), and the MVs, $n_{y}$ is the number of CVs, and $n_{u}$ is the number of MVs. The vectors $u_{j_{\min }}, \Delta u_{j_{\min }}, y_{j_{\min }}, u_{j_{\max }}, \Delta u_{j_{\max }}$, and $y_{j_{\max }}$ are the minimum and maximum values for the inputs, input changes, and outputs whereas $V_{j_{\min }}^{u}, V_{j_{\min }}^{\Delta u}, V_{j_{\min }}^{y}, V_{j_{\max }}^{u}, V_{j_{\max }}^{\Delta u}$, and $V_{j_{\max }}^{y}$ are the ECR (Equal Concern for Relaxation) vectors that govern how much constraint violations can be tolerated [12]. The default disturbance model provided in the MPC Toolbox was used, which assumes an integrated white noise disturbance on each measured CV.

\subsection{MPC with iterative model linearisation}

The base-case MPC described above is designed for the nominal LTI plant. The fuel gas blending system is nonlinear and base-case MPC performance would be expected to degrade when conditions are far from nominal. There are several established ways to compensate for a nonlinear plant. The most general is to use a nonlinear dynamic plant model in combination with an appropriate nonlinear programming code to perform the optimisation.

In the fuel gas blending application, however, the primary source of nonlinearity is a feed gas composition disturbance, which can cause transfer function gains to change sign. Feed composition disturbances are measurable but cannot be anticipated. Once such a change has occurred, the plant behaves in a near-linear manner.

Thus, the present work adapts the controller's LTI model in response to the measured feed compositions, i.e., every $10 \mathrm{~min}$. Otherwise, the MPC definition remains unchanged from the nominal case, and the standard quadratic programming (QP) approach solves the optimisation problem. This is less computationally intensive than the more general nonlinear MPC approach described above, and avoids the possibility of convergence difficulties, i.e., non-convex nonlinear programming problems with multiple optima.

In initial tests it was observed that for the typical range of fuel demand and feed gas compositions the response times and delays change very little. To a good approximation, it is sufficient to adapt the transfer function gains only. Section 4 describes the way in which these depend on the feed compositions. The dynamic parameters in the base-case transfer functions (the time constants and delays) were held constant. Moreover, all other parameters used to define the base-case MPC were retained in the iterative linearisation case. Thus, observed differences are due to the impact of iterative linearisation only.

\subsection{Results}

In all simulations, the nonlinear state-space model described in Section 3 represented the real fuel-gas blending process. Two percent peak-to-peak variability was assumed in the fuel gas demand but for simplicity, actuator dynamics were neglected, perfect flow manipulation was assumed for the MVs, and CV and composition measurements were assumed noise-free.

In the test scenario, two demand disturbances (changes in the total discharge) occur at times 1 and $3 \mathrm{~h}$ respectively, each of a $3 \mathrm{kN} \mathrm{m}^{3} / \mathrm{h}$ magnitude (see Fig. 5). A step disturbance in the NG feed occurs at time $2 \mathrm{~h}$, changing its composition from $\left[\mathrm{CH}_{4}, \mathrm{C} 2+\right.$, $\left.\mathrm{H}_{2}, \mathrm{~N}_{2}, \mathrm{CO}, \mathrm{CO}_{2}\right]=[0.911,0.068,0.0,0.015,0.0,0.006]$ to $[0.841$,

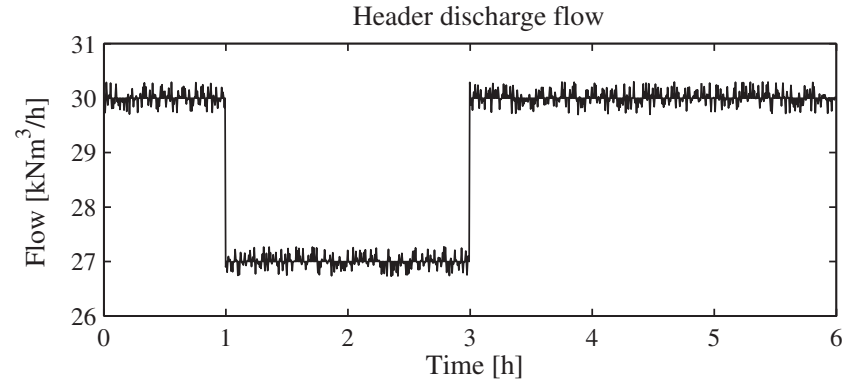

Fig. 5. Header discharge flow.

$0.088,0.01,0.035,0.01,0.016]$. The composition of Tail Gas 1 changes at time $4 \mathrm{~h}$ from $\left[\mathrm{CH}_{4}, \mathrm{C} 2+, \mathrm{H}_{2}, \mathrm{~N}_{2}, \mathrm{CO}, \mathrm{CO}_{2}\right]=[0.055$, $0.01,0.62,0.025,0.26,0.03]$ to $[0.075,0.04,0.57,0.025,0.26,0.03]$. This changes the HHV of $\mathrm{TG}_{1}$ from 13.98 to 17.98 which causes the gain of the model from $\mathrm{TG}_{1}$ to $\mathrm{HHV}$ to change sign (from negative to positive).

Figs. 6-8 compare the performance of the base-case MPC to that using iterative linearisation. For the most part, performance is similar. Fuel gas demand changes and the NG composition disturbance are handled easily. Both controllers stay within the defined CV constraints at all times. This is surprising for the base-case MPC, for which the nominal LTI model becomes inaccurate when the first composition disturbance occurs at time $2 \mathrm{~h}$. In fact, the base-case MPC remains stable with reasonable performance even when one of its transfer function gains has an incorrect sign (from hour 4 onward). Recall that the gain in question is the one relating the Tail Gas $1 \mathrm{MV}$ to the HHV CV.

The explanation is that the base case MPC predicts (incorrectly) that the use of Tail Gas 1 would decrease the HHV, and thus uses natural gas instead, holding Tail Gas 1 at zero flow. In contrast, the MPC with iterative linearisation uses significant amounts of both tail gases from hour 4 onward. If other circumstances had forced the base case MPC to increase the Tail Gas 1 rate during this time, a serious prediction error would have occurred, likely leading to a constraint violation.
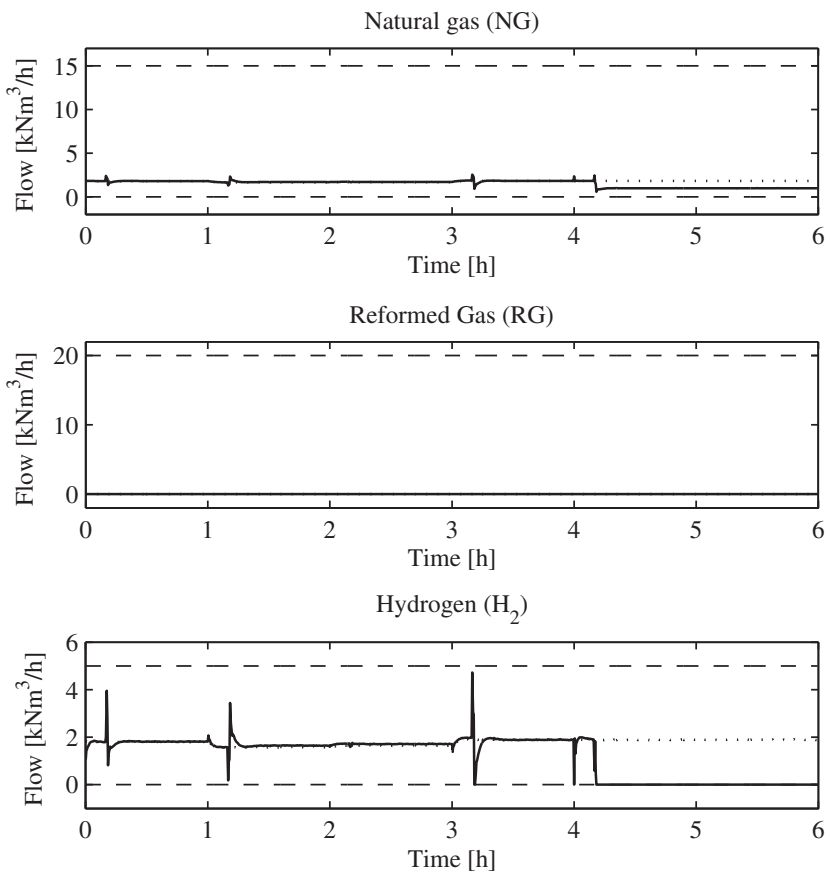

Fig. 6. $\mathrm{NG}, \mathrm{RG}$, and $\mathrm{H}_{2}$ flows for the constant LTI model (dotted) versus using iterative linearisation (solid). The dashed lines indicate the limits. 


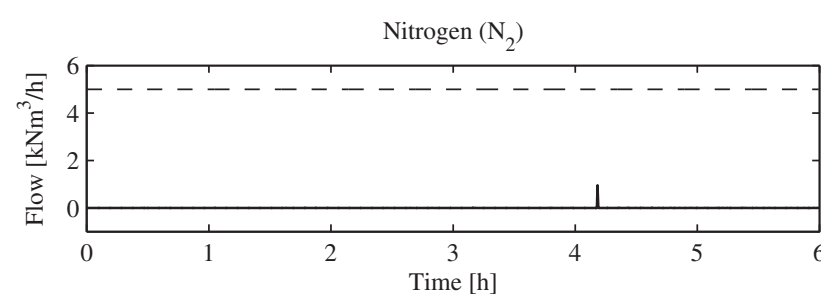

Tail Gas $1\left(\mathrm{TG}_{1}\right)$

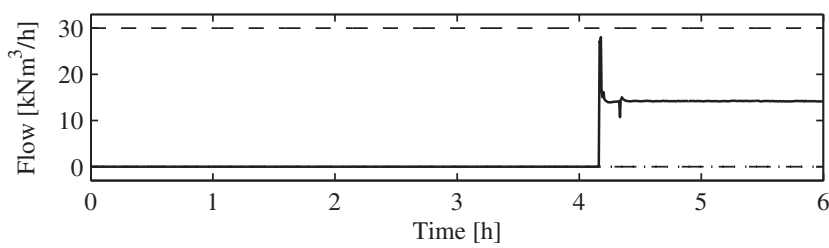

Tail Gas $2\left(\mathrm{TG}_{2}\right)$

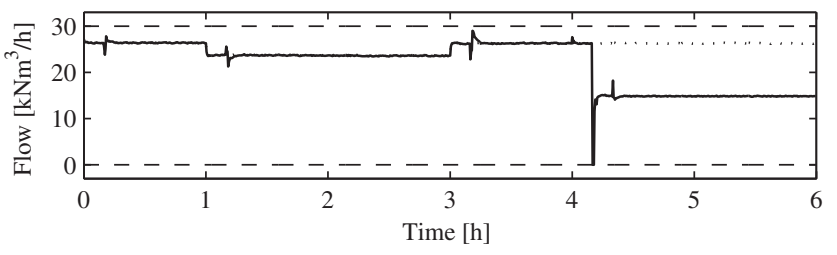

Fig. 7. $\mathrm{N}_{2}, \mathrm{TG}_{1}$, and $\mathrm{TG}_{2}$ flows for the constant LTI model (dotted) versus using iterative linearisation (solid). The dashed lines indicate the limits.

The primary advantage of iterative linearisation in this test appears in the operating costs (calculated from the flow rates and normalised costs), which are shown in Fig. 9. For perspective, the optimal steady-state cost for the time-varying feed compositions is included.

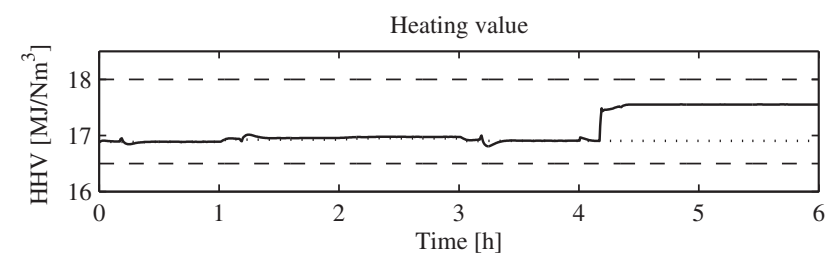

Wobbe index

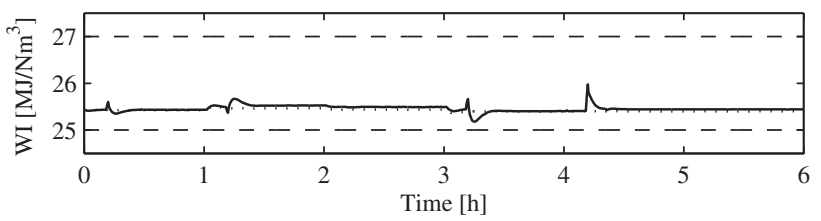

Flame speed

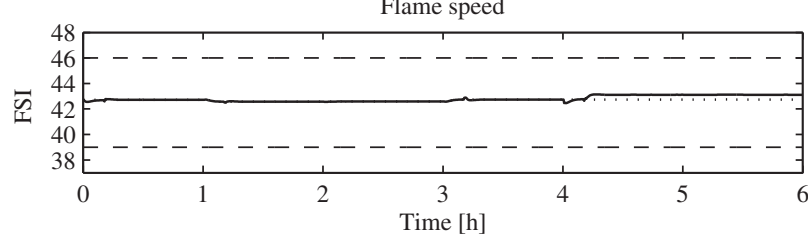

Header pressure

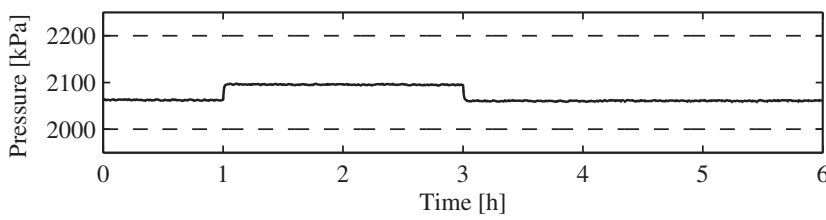

Fig. 8. Controlled variables for the constant LTI model (dotted) versus using iterative linearisation (solid). The dashed lines indicate the limits.

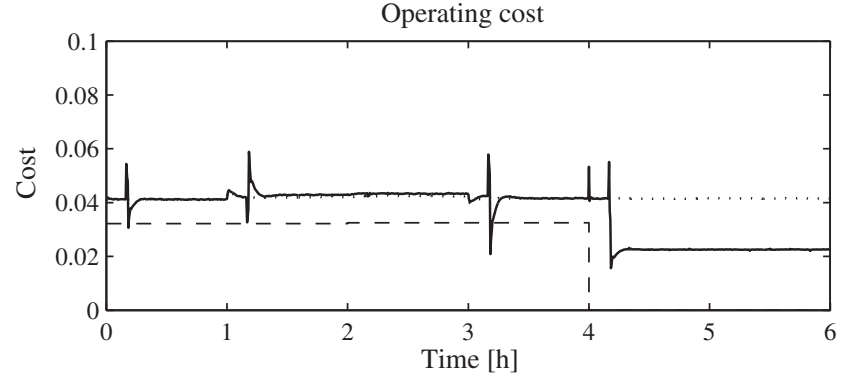

Fig. 9. Operating cost and ideal optimal cost (dashed line) for the constant LTI model (dotted) versus using iterative linearisation (solid).

The cost plots show that a rather small feed composition disturbance can cause a significant change in the optimal strategy. For example, the Tail Gas $1 \mathrm{HHV}$ increase at $4 \mathrm{~h}$ would, in theory, allow the NG feed to be turned off, reducing the fuel gas cost to zero.

Neither controller achieves this, however. For MPC with iterative linearisation the total cost (i.e., the operating cost integrated over the 6 -h test) is $0.2172,13.1 \%$ less than for base-case MPC, but well above the optimal cost of 0.1294 . Considering only the period after the composition change at $4 \mathrm{~h}$, the cost for MPC with iterative linearisation is 0.0225 per $\mathrm{kN} \mathrm{m}^{3}$, a reduction of $45.8 \%$ relative to the base case, but well above the true value, 0 .

This is due to the optimisation problem formulation used in these two controllers. It is difficult to determine controller parameters that provide adequate transient performance (e.g., no constraint violations) while also minimising the steady-state cost. The mid-range CV targets with non-zero CV penalty weights, and the constant MV targets are examples of parameters necessary for adequate dynamic performance but these also limit the controller's ability to reach the theoretical optimal cost. The next section provides a solution to this problem by introducing real-time steady-state optimisation (RTO).

\subsection{Real-time optimisation (RTO)}

In order to push the operating cost towards the true minimum as operating conditions change, an online economic optimisation technique is employed in concert with MPC. This is often termed real-time optimisation (RTO) [14-16].

Most RTO applications involve the use of nonlinear models containing uncertain parameters that must be estimated in order to determine the optimum operating condition [14,17]. In the gas blending case, however, the economic cost is a linear function of the MVs with known, constant coefficients, and model predictions can be performed effectively using iterative linearisation, as described above.

Thus, a straightforward extension of the original MPC strategy is employed to incorporate RTO. Each time a feed composition measurement becomes available (every $10 \mathrm{~min}$ ), a nonlinear program (NLP) is solved to determine the optimal steady-state MV and CV targets. The NLP cost function is linear (convex) but some constraints are non-convex, making it impossible to guarantee a unique solution. No instances of multiple optima have been observed in this application, however.

The calculated CV and MV targets are employed in the MPC cost function (recall that these targets were constant for the test simulations reported above). Otherwise, the controller tuning remains unchanged, preserving the original transient performance.

Figs. 10-13 show the results (for the iterative linearisation version only). For the 6 -h test, the total cost drops from 0.2172 to 0.1369 (a reduction of $37.0 \%$ ). For perspective, if it were possible to drive the system to the optimum steady-state instantly follow- 

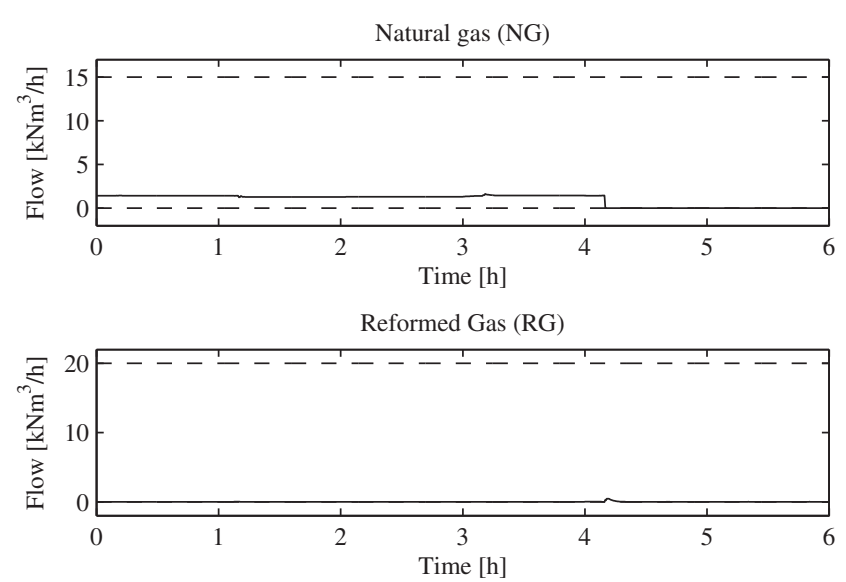

Hydrogen $\left(\mathrm{H}_{2}\right)$

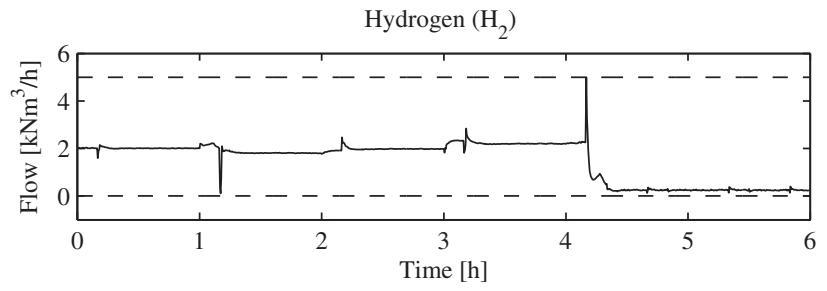

Fig. 10. NG, RG, and $\mathrm{H}_{2}$ flows with RTO. The solid lines are the MV values with the dashed lines indicating the limits.

ing each feed composition disturbance, the cost would have been 0.129 . Thus, the introduction of RTO enables operation close to the optimal cost.

This is also evident from Fig. 13, which shows that the controller eventually reaches the optimal steady-state cost in all cases. The primary cause of sub-optimal performance is the delay in the feed composition measurements, which causes the CV and MV targets to be sub-optimal from $4 \mathrm{~h}$ until the next feed composition measurement becomes available. This suggests that there would be little to

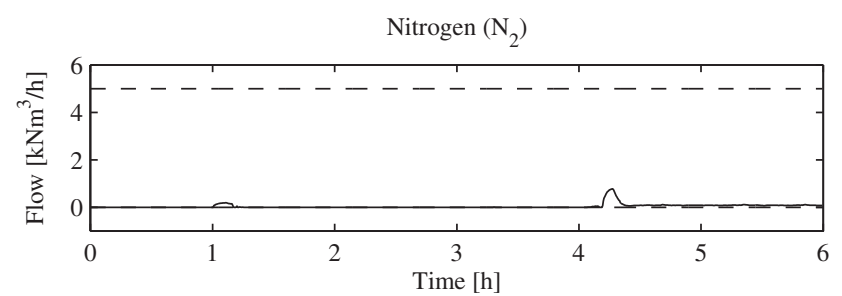

Tail Gas $1\left(\mathrm{TG}_{1}\right)$

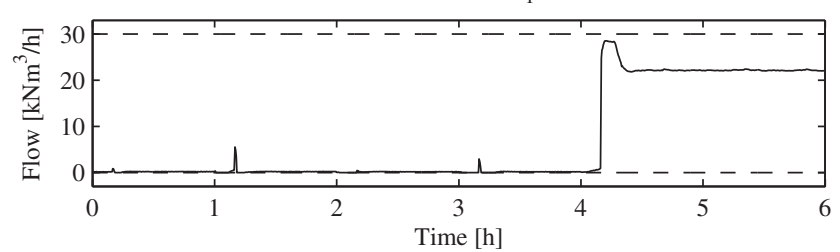

Tail Gas $2\left(\mathrm{TG}_{2}\right)$

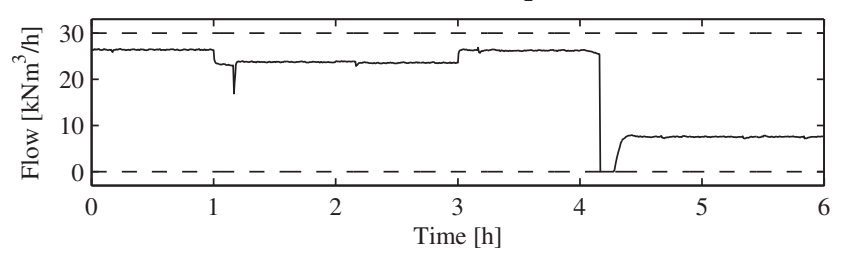

Fig. 11. $\mathrm{N}_{2}, \mathrm{TG}_{1}$, and $\mathrm{TG}_{2}$ flows with RTO. The solid lines are the MV values with the dashed lines indicating the limits.

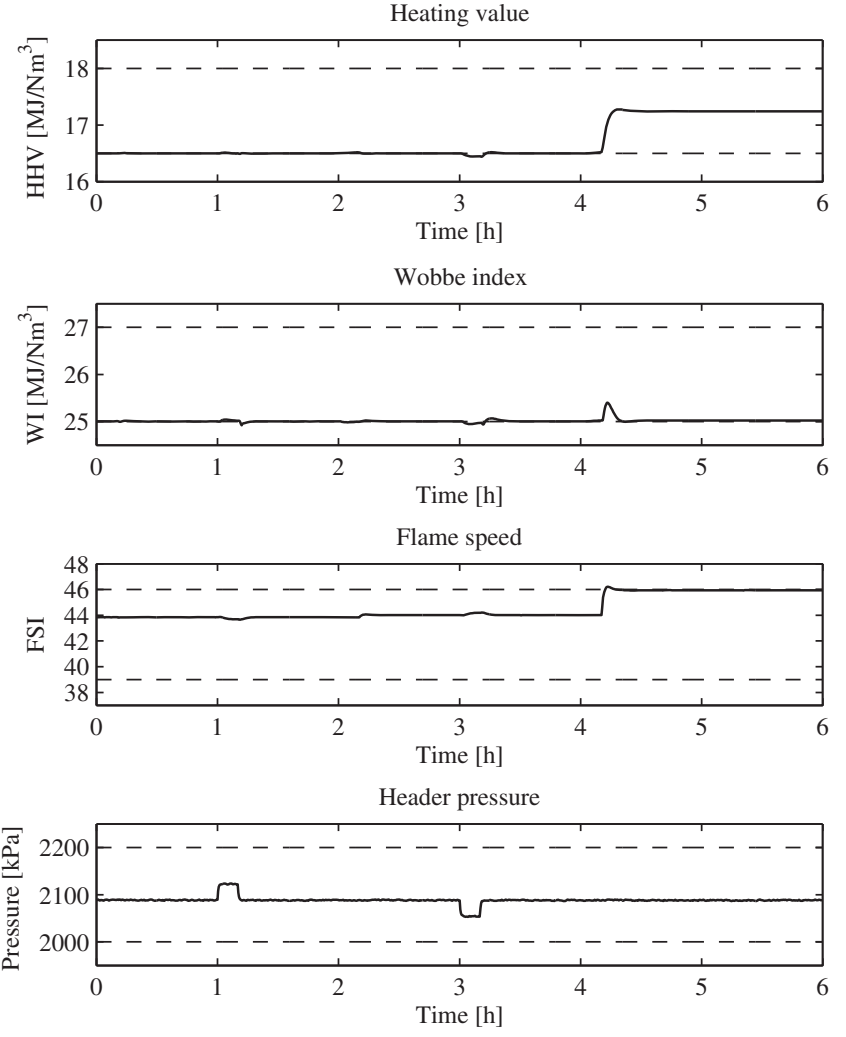

Fig. 12. Controlled variables with RTO. The solid lines are the CV values with the dashed lines indicating the limits.

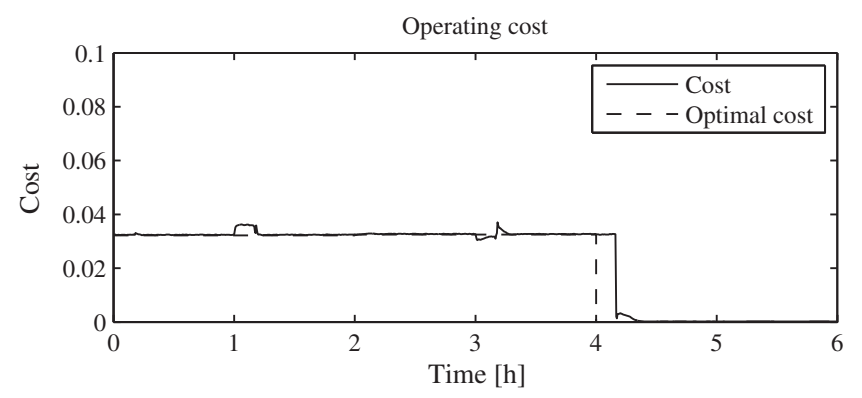

Fig. 13. Operating cost and ideal optimal cost (dashed line) with RTO.

gain through the use of a more general dynamic RTO strategy. This remains as a possibility for future research.

From Fig. 12, it is clear that transient performance is still excellent with negligible constraint violations, even though the HHV, WI, and FSI have been driven to bounds for most of the 6-h test (the header pressure has no impact on the cost, so it stays near its constant mid-range target).

Table 10 summarises and compares the average costs per hour interval for the three cases and the optimal steady-state cost. These

Table 10

Time averaged costs per $1 \mathrm{~h}$ interval for the constant LTI case, the iterative linearisation case, the RTO case, and the steady-state optimal values.

\begin{tabular}{lllll}
\hline Interval & Const. LTI & Iter. Lin. & RTO & SS Opt. \\
\hline 0-1 h & 0.0411 & 0.0411 & 0.0324 & 0.0322 \\
$1-2 \mathrm{~h}$ & 0.0422 & 0.0430 & 0.0330 & 0.0322 \\
2-3 h & 0.0425 & 0.0433 & 0.0327 & 0.0325 \\
3-4 h & 0.0415 & 0.0413 & 0.0326 & 0.0325 \\
$4-5 \mathrm{~h}$ & 0.0415 & 0.0259 & 0.0060 & 0.0000 \\
$5-6 \mathrm{~h}$ & 0.0415 & 0.0225 & 0.0002 & 0.0000 \\
\hline
\end{tabular}


values are calculated by adding the cost values in each one hour interval and dividing by the number of samples in the interval.

\section{Conclusion}

The proposed first-principle nonlinear state-space model for the fuel gas blending process provides an adequate representation of the real industrial plant. The correlations between simulation and plant data indicate that the key assumptions (ideal gas, uniform mixing) are reasonable. Therefore, the simulation allows realistic tests of advanced control and optimisation strategies. The fuel gas blending application is similar to other blending operations described in the literature, but it offers unique dynamic and economic characteristics.

The use of iterative linearisation (a form of nonlinear MPC) provides excellent compensation for the fuel gas blending system's nonlinear characteristics. The four CVs are regulated well despite feed gas disturbances and fuel gas demand variations. This is the case even when one or more CVs is driven to a boundary in order to achieve minimum-cost operation. There are minor-short term violations of the soft CV constraints, but these would be acceptable in the real plant. If this were not the case, safety margins could be imposed but this would increase the operating cost.

When RTO is used to update long-term CV and MV targets, MPC (with iterative linearisation) drives the plant reliably to the optimal steady-state condition following a disturbance. Although there is potential for even better economic performance, the results suggest that the primary limiting factor is the measurement system, not the controller. Thus, it is unlikely that a more general nonlinear dynamic RTO approach would be justified. Whether this is indeed the case is a possible area for future research.

\section{Acknowledgements}

Thanks to Adolf Wolmarans from Sasol Infrachem for giving permission to use the plant data and to Paul Hughes from Sasol
Technology for his advice and guidance (especially with regard to the iterative linearisation).

\section{References}

[1] D.W. Green, et al., Perry's Chemical Engineers' Handbook, Seventh Edition, McGraw-Hill, 1997, pp. 2-7-2-44.

[2] F. Johnson, D.M. Rue, Gas Interchangeability Tests: Evaluating the Range of Interchangeability of Vaporized LNG and Natural Gas, Gas Technology Institute for Gas Research Institute, April 2003.

[3] M. Chèbre, Y. Creff, N. Petit, Feedback control and optimization for the production of commercial fuels by blending, J. Process Control 20 (4) (2010) 441-451.

[4] P. Hughes, Sasol Fuel Gas Optimiser: APC Controller Feasibility Study, Sasol Technology, Sasolburg, May 2008.

[5] P. Hughes, SCI Fuel Gas Optimiser: Engineering Documentation, Sasolburg, Sasol Technology, March 2010, pp. 30-32.

[6] P. Hughes, Discussion on the derivatives of HHV, WI, and FSI, Personal communication, Sasol Technology, Sasolburg, June 2010.

[7] J. Richalet, A. Rault, J.L. Testud, J. Papon, Algorithmic control of industrial processes, in: IFAC, Proc. 4th IFAC Symp. Identif. Syst. Parameter Estim., 1976, pp. 1119-1167.

[8] C.R. Cutler, B.L. Ramaker, Dynamic matrix control-a computer control algorithm, in: Proc. Jt. Auto. Control Conf., San Francisco, 1980.

[9] J.B. Rawlings, Tutorial Overview of Model Predictive Control, IEEE Control Syst. Mag. 20 (3) (June 2000) 38-52.

[10] E.F. Camacho, C. Bordons, Model Predictive Control, Second Edition, Springer, London, 2007, pp. 1-10.

[11] S.J. Qin, T.A. Badgwell, A survey of industrial model predictive control technology, Control Eng. Pract. 11 (July 7) (2003) 733-764.

[12] A. Bemporad, M. Morari, N.L. Ricker, Model Predictive Control Toolbox 3: User's guide, The MathWorks, Inc., 2010.

[13] D.E. Seborg, T.F. Edgar, D.A. Mellichamp, Process Dynamics and Control, Second Edition, Wiley, NJ, 2004.

[14] V. Adetola, M. Guay, Integration of real-time optimization and model predictive control, J. Process Control 20 (2) (2010) 125-133.

[15] J.H. Lee, J.M. Lee, T. Tosukhowong, J. Lu, On interfacing model predictive controllers with a real-time optimizer, Comput. Aided Chem. Eng. 15 (C) (2003) 910-915.

[16] C.R. Cutler, R.T. Perry, Real time optimization with multivariable control is required to maximize profits, Comput. Chem. Eng. 7 (February 5) (1983) 663-667.

[17] G. De Souza, D. Odloak, A.C. Zanin, Real time optimization (RTO) with model predictive control (MPC), Comput. Chem. Eng. 34 (July 12) (2010) 1999-2006. 